\title{
YORP effect on real objects
}

\section{Statistical properties}

\author{
M. Micheli ${ }^{1,2}$ and P. Paolicchi ${ }^{1}$ \\ 1 Dipartimento di Fisica, Università di Pisa, Largo Pontecorvo 3, 56127 Pisa, Italy \\ e-mail: paolicchi@df.unipi.it \\ 2 Institute for Astronomy, University of Hawaii, 2680 Woodlawn Drive, HI-96822 Honolulu, USA \\ e-mail: micheli@ifa.hawaii.edu
}

Received 25 October 2007 / Accepted 15 July 2008

ABSTRACT

\begin{abstract}
Context. The intensity of the YORP (Yarkovsky, O'Keefe, Radzievskii, and Paddack) effect and its ability to affect the rotational properties of asteroids depend mainly on the size of the body and on its shape. At present, we have a database of about 30 welldefined shapes of real minor bodies (most of them asteroids, but also planetary satellites and cometary nuclei).

Aims. In this paper we perform a statistical analysis of how the YORP effect depends on the shape.

Methods. We used the Rubincam approximation (i.e. neglecting the effects of a finite thermal conductivity).

Results. We show that, among real bodies, the distribution of the YORP types, according to the classification of Vokrouhlický and Čapek, is significantly different from the one obtained in the same paper from theoretical modeling of shapes. A new "type" also comes out. Moreover, we show that the types are strongly correlated with the intensity of the YORP effect (when normalized to eliminate the dependence on the size, and thus only related to the shape).
\end{abstract}

Key words. minor planets, asteroids

\section{Introduction}

In recent years, the relevance of the YORP effect for the rotational properties of the asteroids has been widely discussed (see, for instance, Vokrouhlický et al. 2003). Also its possible indirect implications for the dynamical evolution of asteroids have been put into evidence, mainly for what concerns the members of asteroid families (Vokrouhlický et al. 2003, 2006). In spite of its complex dependence on the physical properties of the bodies and of being entangled with other dynamical effects (such as spin-orbit coupling, secular resonances, and so on; e.g., Bottke et al. 2006), the YORP effect has recently become a major player in the overall modeling of the evolution of asteroids and of asteroid families, as well as of dynamical transfer processes from the Main Belt to the NEAs region. Even more recently, two direct observational proofs of the effect have been obtained (Kaasalainen et al. 2007; Lowry et al. 2007; Taylor et al. 2007).

Theoretical models have been introduced that either consider or neglect the effects of a finite thermal conductivity (Rubincam 2000; Vokrouhlický \& Čapek 2002; Čapek \& Vokrouhlický 2004) and also try to analyze the effect on an artificial sample of model bodies (again in Vokrouhlický \& Čapek 2002), in order to understand how the effect depends on the shape of the bodies. Recently, Nesvorný \& Vokrouhlický $(2007,2008)$ have developed the first completely analytic theory of the phenomenon that is able, under the assumption of a near-spherical shape, to predict some of the key aspects of the YORP effect. In general, no easy intuitive readout of the results is possible. However, a rough taxonomy of the behavior of bodies with regard to the YORP effect has been introduced in Vokrouhlický \& Čapek (2002); four main classes of objects have been defined, and the above-quoted papers by Nesvorný \& Vokrouhlický $(2007,2008)$ provided a first analytical interpretation of their origin.

In recent times the number of minor bodies whose shape is known has increased widely. In the present paper, we first present a systematic analysis of the effect on all the minor bodies for which the shape data are significant enough. In this way, we can obtain a first estimate of the frequency of different taxonomic (in YORP sense) classes among real bodies. Moreover, we have found a statistically significant correlation between the YORP type and the intensity of the effect, whose meaning and implications we discuss in detail.

After this statistical analysis of our results, a detailed description of the effect on all the individual bodies in the sample will be presented in the second paper of this series (in preparation).

\section{Data and methods}

The sample used for our analysis was obtained by collecting three-dimensional shape models available in the literature.

In our sample we then have 30 objects, as listed in Table 1; among them 19 asteroids, 1 cometary nucleus, and 10 natural satellites of major planets. We decided to include non-asteroidal models in the sample, since we do not find any a priori reason why cometary nuclei or satellites should have a systematically different shape. However, our analysis will also take the sample limited to the asteroids into account, to verify this assumption a posteriori and to avoid possible biases.

Most of the models that we use were obtained by means of a delay-Doppler radar imaging, while others were obtained by a three-dimensional model obtained from space missions. 
Table 1. List of the objects studied in this work.

\begin{tabular}{cc}
\hline \hline Asteroids \\
\hline \hline Object & YORP type \\
\hline (4) Vesta & 3 \\
(216) Kleopatra & 2 \\
(243) Ida & 1 \\
(253) Mathilde & 3 \\
(433) Eros & 1 \\
(951) Gaspra & 1 \\
(1580) Betulia & 1 \\
(1620) Geographos & 2 \\
(2063) Bacchus & 4 \\
(4179) Toutatis & 1 \\
(4769) Castalia & 3 \\
(6489) Golevka & 4 \\
(10115) 1992 SK & 1 \\
(25143) Itokawa & 1 \\
(29075) 1950 DA & $1-4$ \\
(52760) 1998 ML14 & 1 \\
(66391) 1999 KW4 & 4 \\
S/2001 (66391) 1 & 1 \\
1998 KY26 & 1 \\
\hline Cometary nuclei & \\
\hline Object & YORP type \\
\hline \hline 1P/Halley & 1 \\
\hline Planetary satellites & \\
\hline Object & YORP type \\
\hline Maturn I (Phobos) & 4 \\
Saturn XVII (Epimetheus) & 2 \\
Neptune VII (Larissa) & 2 \\
Neptune VIII (Proteus) & 4 \\
\hline Jupiter V (Amalthea) & 3 \\
Saturn VII (Hyperion) & 2 \\
\hline \hline \\
\hline \hline
\end{tabular}

All the cometary and the satellite models have been obtained with the latter method. The data were taken from a few web-databases. Some radar models come from the cata$\log$ by Neese et al. (2004), downloaded from http://www . psi.edu/pds/resource/rshape.html, while others come from http://echo.jpl.nasa.gov/links.html. The optical models were obtained from the databases by Neese \& Thomas (2000) and Neese \& Stooke (2002), and can be downloaded from http: //WWW . psi . edu/pds/resource/oshape. html and http: //wWw.psi.edu/pds/resource/stkshape. html.

\subsection{Lightcurve-inversion models}

We included all the available models from radar and spacecraft observations, but none of those obtained with lightcurveinversion methods. These are unable to represent the surface concavities in detail (see, for instance, Durech 2002, and references therein). We think that this limitation might introduce significant biases into the computations concerning the YORP effect.

To test this assumption we analyzed some of the lightcurve models published in http://astro.troja.mff.cuni.cz/ projects/asteroids3D.
The first test aimed to directly verify whether the most prominent property of a lightcurve-inversion model, its imposed convexity, influences the YORP effect; we downloaded the models of the only two objects for which the above-quoted website provides both a convex and a non-convex shape, namely (1627) Ivar and (3908) Nyx. If the lightcurve-inversion models were usable for a YORP analysis, convex and non-convex models should provide similar results (at least in terms of the taxonomic classification). Indeed, (1627) Ivar results in a "type 2" in the convex version and a "type 1" in the non-convex one (for a discussion of the taxonomic types, see Vokrouhlický \& Čapek 2002), while (3908) Nyx changes from a "type 3" to a "type 1". Either the non-convex models are accurate enough to test the YORP, while the convex ones are not (and in this case none of the other convex lightcurve-inversion models is acceptable), or both the models are too imprecise for our YORP purposes (because the smallscale features wiped out in these models are relevant).

The second test involved the use of the convex lightcurveinversion shape of (1580) Betulia, for which we also have the radar model. In this case the object, a standard "type 1" from the radar version, becomes a new and complex YORP type (that we might define as a "type 6", being the mirror case of the "type 5" defined below, and represented in Fig. 1) using the lightcurveinversion shape.

In our opinion, both these results entail the exclusion of the asteroidal models obtained from lightcurve-inversion procedures from our analysis, and it is not easy to decide if and when the resulting shape model may be meaningful. Moreover, these results confirm the extreme sensitivity of the YORP effect to the details of the shape (in agreement with the discussion of Nesvorný \& Vokrouhlický 2007). All the YORP results obtained so far, including the ones presented below, should always be seen and considered under this perspective.

\subsection{Modeling}

To compute the YORP effect we used a polyhedron model with triangular facets, as in Vokrouhlický \& Čapek (2002). In a few cases, the model was already available in the literature. In other cases it was reconstructed from the data by adopting a resolution corresponding to about 1200 facets, which appears a good compromise between the need to save computer time and the achievement of the most reliable results that can be obtained from the available data. The three-dimensional model has always been assumed to rotate around the maximum inertia axis. We adopted, for sake of simplicity, the approximation of zero thermal conductivity, as done by Rubincam (2000). For some objects (especially those observed during spacecraft fly-bys), the data are not complete, meaning the YORP model may also not be very accurate. Moreover, asteroid (29075) 1950 DA can be reconstructed (Busch et al. 2007) using two different assumptions on the orientation of the spin axis, both equally consistent with the data. Two different models come out, and we included both of them in our analysis ${ }^{1}$.

In the plots presented in this series of papers, the YORP torque $\tau$ is normalized to the momentum of inertia related to the spin axis $C$, thus automatically introducing a rate of change for the rotation rate, at least for what concerns the along-axis component $\tau_{\mathrm{s}}$. We assumed a constant density value of $\rho=$ $2500 \mathrm{~kg} \mathrm{~m}^{-3}$. The assumption is not critical to the qualitative

\footnotetext{
1 We can include them as two independent models or weight them $1 / 2$ each: the qualitative results of the analysis, which we report in the following, are independent of this - somewhat arbitrary - choice.
} 
findings, even if it may affect the estimated rates of change for the rotational parameters. However, in the present paper we intend to perform a statistical analysis of how the effects depend on the shape. For this task it may be useful to scale the effect in order to eliminate the dependence on the effective radius $R_{0}$ of the body. The torque caused by the radiation is, for every surface element, (being $\hat{N}$ its normal unit vector) $\mathrm{d} \tau=\boldsymbol{r} \times \boldsymbol{f} \mathrm{d} A$, where

$\boldsymbol{f}=-\frac{2 S}{3 c} \hat{N}=-\frac{2\left(\hat{r_{\mathrm{S}}} \cdot \hat{N}\right) F_{\mathrm{S}}}{3 c} \hat{N}$

according to the Rubincam approximation (and using the same notations as defined in Rubincam 2000): thus $\tau \sim R_{0}{ }^{3}$. Moreover $C \sim M \cdot R_{0}{ }^{2}$ and $M \sim R_{0}{ }^{3}$, then $C \sim R_{0}{ }^{5}$. We obtain $\frac{\tau}{C} \sim \frac{R_{0}{ }^{3}}{R_{0}{ }^{5}} \sim$ $R_{0}{ }^{-2}$.

With the previous scaling rule, we can compare the intensity of the effect among bodies of different shapes, eliminating every size dependence. To do so, we also have to define a "mean value" of the YORP torque $\tau$, for a given body, which depends on the inclination of the spin axis $(\Theta)$. We assumed the rms. variance of the function $\tau(\Theta)\left(0 \leq \Theta \leq 180^{\circ}\right)$ as the amplitude of each component; then we summed up the two significant squared components $\tau_{\Theta}$ and $\tau_{\mathrm{s}}$. (The third component $\tau_{\psi}$ is never considered in YORP problems, because it causes only a precession of the rotation axis, not relevant for the spin dynamics.) The resulting value was multiplied per the scaling factor $R_{0}^{2}$. Finally we obtained a quantity $(\tilde{\tau})$ independent on the size of the object. Obviously we refer here only to a direct dependence. Maybe the size also influences the shape, but we do not intend to eliminate this indirect dependence, if there is any.

\section{Results}

A few interesting results come out of the analysis of the previously described database of real shapes.

\subsection{Taxonomy of YORP models}

According to the suggestion by Vokrouhlický \& Čapek (2002), we classified all the objects analyzed in the present study, using the behavior of the component $\tau_{\Theta}$ to include them in the 4 taxonomic types defined there. We added another class "type 5 " to take into account the existence of a model qualitatively different from those identified in the quoted paper. In fact, the satellite Saturn XVII (Pandora) exhibits a new kind of YORP model (see Fig. 1). The function $\tau_{\Theta}$ exhibits two zeros within the range from 0 to $90^{\circ}$, different from all the real objects presented by Vokrouhlický \& Čapek (2002), but consistent with some synthetic models analyzed in the same paper. As discussed in Nesvorný \& Vokrouhlický $(2007,2008)$, the existence of several types is a natural consequence of the dominance of different "modes".

\subsection{Taxonomy: statistical analysis of real objects}

In the above paper (Vokrouhlický \& Čapek 2002), 500 synthetic objects have been built, following the random Gaussian sphere method described by Muinonen (1998), and the resulting YORP model is computed. Starting from this sample, a distribution of objects in the various taxonomic classes is suggested (see the second column in Table 2, taken from Vokrouhlický \& Čapek 2002). In the same table we report also the number of objects from the present analysis pertaining to each class, both using the

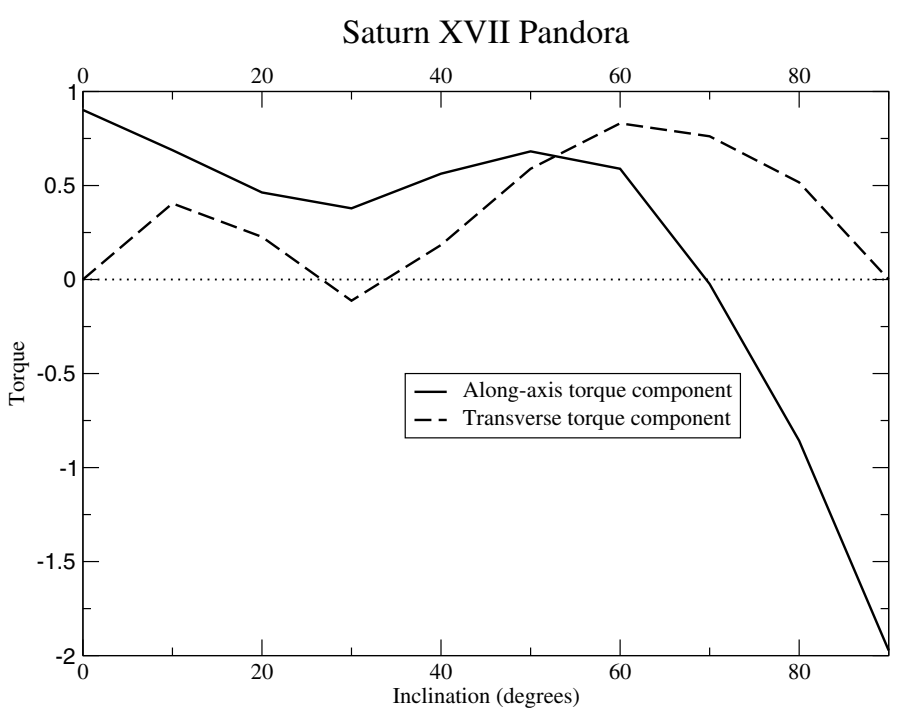

Fig. 1. Plot of the YORP-torque components $\tau_{\Theta}(\Theta) / C$ (dashed curve) and $\tau_{\mathrm{s}}(\Theta) / C$ (solid curve), in units of $10^{-23} \mathrm{~s}^{-2}$ for the satellite Saturn XVII (Pandora).

Table 2. "Theoretical" fraction and the real occurrences pertaining to every class.

\begin{tabular}{cccc}
\hline \hline Type & $p_{i}$ & $N_{i, \mathrm{C}}$ & $N_{i, \mathrm{~A}}$ \\
\hline 1 & 0.392 & 12.5 & 10.5 \\
2 & 0.404 & 6 & 2 \\
3 & 0.102 & 4 & 3 \\
4 & 0.062 & 6.5 & 3.5 \\
5 & 0.040 & 1 & 0 \\
\hline
\end{tabular}

complete sample (third column) and the sample containing only asteroids (fourth column) ${ }^{2}$.

We tested the consistency between our "real" distribution and the theoretical one. We used a statistical approach similar to the usual $\chi^{2}$ test, as for instance in Vokrouhlický et al. (2006). The test parameter is defined as

$\Psi=\sum_{i=1}^{5} \frac{\left(N_{i}-N p_{i}\right)^{2}}{N p_{i}}$

where $p_{i}$ is the relative frequency of the type $i$ in the theoretical model, while $N_{i}$ is the number of real objects in the class and $N=$ $\sum N_{i}$. The parameter has been computed for the real data $\left(\Psi_{\mathrm{r}}\right)$ and also, for comparison, for artificial random samples obtained starting from the theoretical distribution $\left(\Psi_{\mathrm{a}}\right)$.

A significance level $\alpha$ is introduced (usually $1-\alpha=0.95$, i.e. we require a $5 \%$ significance), and we compare the fraction $t$ of artificial cases for which $\Psi_{\mathrm{a}} \geq \Psi_{\mathrm{r}}$ with $\alpha$. We can define a "confidence level" $1-\alpha$ to reject the assumption that the theoretical and the real distributions are identical. The statistical argument takes t he number of real bodies into account, so the result should not depend on the moderate number of objects. The values we obtain are $t_{\mathrm{C}}=0.009<\alpha$, for the complete sample, and $t_{\mathrm{A}}=0.027<\alpha$ for the asteroidal sample. In both cases the theoretical distribution proposed by Vokrouhlický \& Čapek (2002) is significantly different from the real ones.

Another possible test may be to employ fewer classes, collecting the objects whose YORP model is different only in terms

\footnotetext{
2 The two models of the asteroid (29075) 1950 DA have been given each a weight 0.5 .
} 
Size dependence and taxonomy

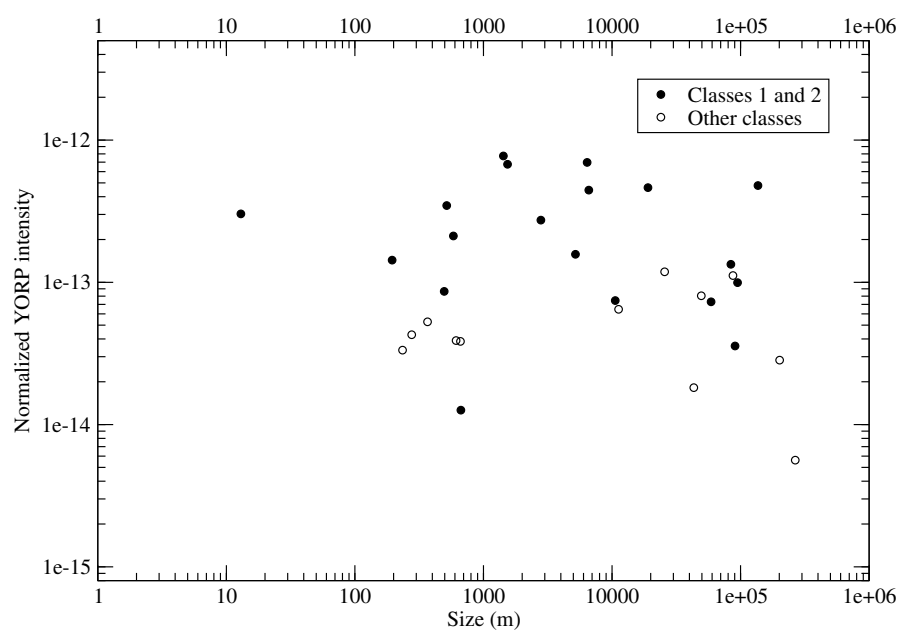

Fig. 2. Logarithmic plot of the normalized YORP intensity $\tilde{\tau}$ (in units of $\mathrm{m}^{2} \mathrm{~s}^{-2}$ ) as a function of the effective radius $R_{0}$. The full circles refer to objects in the classes 1 and 2; the empty circles refer to the other types.

of "parity": types 1 and 2 are collected together, and the same is done for the classes 3 and 4 . The division into classes depends essentially on the number of nodes. The rationale of this approach is based on the assumption of symmetry vs. inversion of the spin axis. In this case, we obtain $t_{\mathrm{C}}^{\prime}=0.022<\alpha$ and $t_{\mathrm{A}}^{\prime}=0.074 \gtrsim \alpha$ : a full rejection in the former case, a marginal acceptance in the latter, maybe due to having fewer objects and classes. It does not seem enough to falsify our qualitative result, i.e. that shapes of real objects are not completely random, at least for what concerns the YORP model. The impossibility of explaining the asteroidal shapes in terms of purely stochastic processes has already been claimed in the literature (see, for instance, La Spina \& Paolicchi 1996). However, this is a completely different argument.

\subsection{The intensity-taxonomy correlation}

The computed $\tilde{\tau}$ values are distributed - within an order of magnitude - around a mean value $\overline{\tilde{\tau}} \simeq 10^{-13} \mathrm{~m}^{2} \mathrm{~s}^{-2}$.

The values appear to be strongly correlated to the YORPtaxonomic type, as shown in Fig. 2. The normalized YORP effect is generally - and significantly - larger for bodies in classes 1 and 2. The bodies in the other classes always have $\tilde{\tau}<1.2 \times$ $10^{-13} \mathrm{~m}^{2} \mathrm{~s}^{-2}$.

In spite of the already claimed difficulties explaining the YORP properties in elementary terms, one can suggest a qualitative explanation for this difference: classes 1 and 2 are those for which a "basic" effect is present, due to simple large-scale shape properties. Whenever this basic effect is absent, minor effects are introduced by more complex, but smaller, irregularities. In fact the objects in the classes 3 to 5 , characterized by a complex ("anomalous") $\tau_{\Theta}(\Theta)$ function, usually have shapes that are, at first sight, by far more "regular" than those pertaining to the "normal" YORP types (1 and 2). When the dominant modes are of high order (i.e. the scale of dominant irregularities is small) the exact value of the effect (even the sign) can be computed only with a highly refined model; in such cases, obviously, a detailed computation, in terms of a polyhedron model, should require a far larger number of facets (see, for instance, Nesvorný \& Vokrouhlický 2007). In principle, one should expect, in these
YORP intensity and critical angle

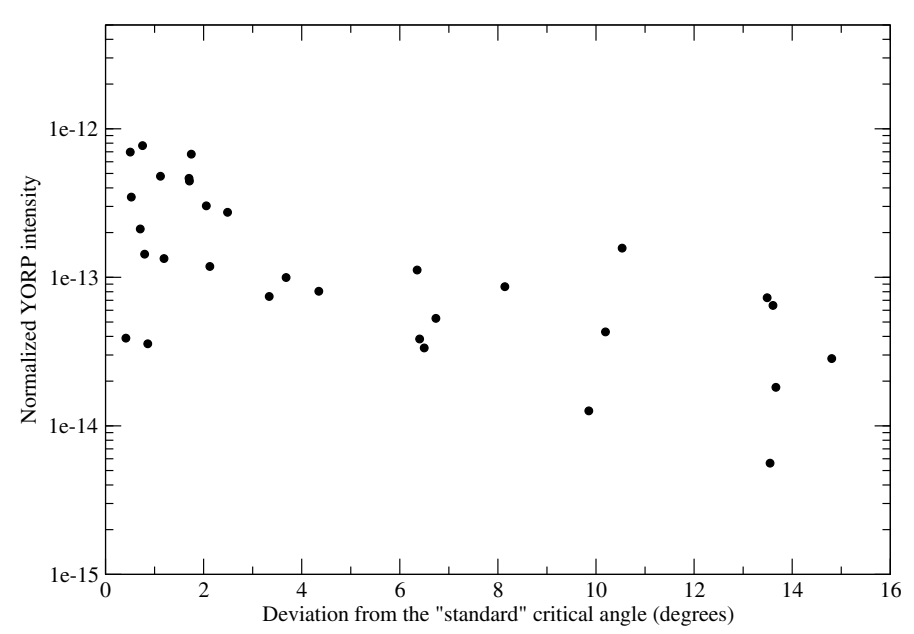

Fig. 3. Normalized YORP intensity $\tilde{\tau}$ as a function of the critical angle.

cases, a weak effect. However, the reality is more complex, as it happens, because the high order terms are dominant in the above quoted case (and to take them into account is needed to fit the observed rate of change of the spin period), but also the low-order terms are not negligible, and the overall (normalized) effect is around the mean values. Even if serious caveats are introduced whenever we wish to assess the behavior of a particular object with the aid of a simplified model, the problem is not critical to our statistical analysis.

\subsection{Discussion of the critical angle}

In the pioneering paper by Rubincam (2000), some seemingly general properties of the YORP effect were made evident. Among them the presence of a critical value for the rotation axis obliquity $\Theta_{c} \simeq 56^{\circ}$, corresponding to a zero of the YORP-torque component along the spin axis, $\tau_{\mathrm{s}}$ was probably the most evident.

Further analysis (see Vokrouhlický \& Čapek 2002) has shown that this property is not very general. In Fig. 3 we plot the absolute value of the difference between the critical angles of various objects (defined as the angle for which $\tau_{\mathrm{s}}=0$ ) and the "reference critical angle" $56^{\circ}$ vs. the normalized intensity of YORP effect (defined above). When more than one zero is present in the function $\tau_{\mathrm{s}}(\Theta)$, we take the angle closer to the reference value.

A strong correlation comes out, with a Spearman rank correlation coefficient (Spearman 1904) $\rho \simeq 0.65$. (It means, for our number of objects, that the correlation is proved with a significance of about $99.992 \%$.) We can conclude that the critical angle close to the reference value is typical of the most intense YORP effects, which, again, one may understand as due to simple largescale irregularities.

We can compute a "mean value" of the critical angle, using the normalized intensity as a weight in the computation. One using the complete sample of real objects to obtain $\overline{\Theta_{c}}=(56.0 \pm$ $0.5)^{\circ}$.

A different way to compute the same value again uses the Spearman rank correlation coefficient, to compute it in the same way as described above, but using a variable value for the reference critical angle. Then we can find which value of that reference angle maximizes the coefficient. In our case this happens for $\Theta_{\mathrm{c}} \simeq 56.7^{\circ}$, where $\rho \simeq 0.69$ and the significance of the 
correlation reaches $99.998 \%$. To provide an error for this new estimation, we can calculate the range of values in which the significance of the correlation is above a chosen level; if we use a confidence limit of $99.99 \%$, we obtain acceptable values of the critical angle in all the interval between $55.8^{\circ}$ and $57.3^{\circ}$.

Therefore we can conclude that the actual "critical angle" is probably somewhere between $56^{\circ}$ and $57^{\circ}$; however, it is not easy to obtain a simple, intuitive explanation of this value. We recall that, in the paper by Vokrouhlický et al. (2003) devoted to the Koronis family, the critical angle has been tentatively connected to a known property of a spherical planet. If the inclination is $\Theta \simeq 54^{\circ}$, the insolation is almost exactly uniform, when taking the mean over a period of revolution. Apart from more complex considerations (the uniformity breaks down completely whenever one introduces even small deviations from the spherical shape), the present computation of the critical YORP-angle, with its small statistical uncertainty, seems to rule out a coincidence between the two numerical values and thus any argument starting from this claimed coincidence. The same result is obtained analytically in Nesvorný \& Vokrouhlický (2007), who show that the critical angle is caused by a complex coupling between the insolation and the longitudinal shape irregularities, and is also dependent on the spectrum of such irregularities.

\section{Conclusions and future work}

In this paper we have analyzed the YORP effect in a significant sample of real minor bodies. We also classified the types of the bodies, according to the taxonomy introduced in Vokrouhlický \& Čapek (2002) on the basis of a sample of artificial shapes. We find that the distribution of types in the classes is different for the sample of real objects, compared to the distribution coming out from the synthetic models. Moreover, we have shown that the "types" correspond to a systematically different intensity of the torque, when normalized to eliminate the dependence on the size. We suggest that a "basic", strong YORP effect acts on bodies with large-scale and simple irregularities (types 1 and 2 ). Whenever the basic effect is absent or reduced, more complex irregularities cause a weaker and less predictable YORP pattern (other types).

A detailed analysis of the individual real cases is also useful, and we will present some of them in the next paper, together with the analysis of how the YORP may affect, with time, the distribution of spin vectors.

Acknowledgements. We are grateful to the Referee David Vokrouhlický for useful comments and suggestions. Paolo Paolicchi has been supported by ASI funds.

\section{References}

Bottke, Jr., W. F., Vokrouhlický, D., Rubincam, D. P., \& Nesvorný, D. 2006, Annu. Rev. Earth Planet. Sci., 34, 157

Busch, M. W., Giorgini, J. D., Ostro, S. J., et al. 2007, Icarus, 190, 608

Kaasalainen, M., Durech, J., Warner, B. D., Krugly, Y. N., \& Gaftonyuk, N. M. 2007, Nature, 446, 420

La Spina, A., \& Paolicchi, P. 1996, Planet. Space Sci., 44, 1563

Lowry, S. C., Fitzsimmons, A., Pravec, P., et al. 2007, Science, 316, 272

Muinonen, K. 1998, A\&A, 332, 1087

Neese, C., \& Stooke, P. 2002, NASA Planetary Data System, EAR-A-5-DDRSTOOKE-SHAPE-MODELS-V1.0

Neese, C., \& Thomas, P. 2000, NASA Planetary Data System, EAR-A-5-DDRSHAPE-MODELS-V2.1

Neese, C., Hudson, S., \& Ostro, S. 2004, NASA Planetary Data System, EARA-5-DDR-RADARSHAPE-MODELS-V2.0, 16

Nesvorný, D., \& Vokrouhlický, D. 2007, AJ, 134, 1750

Nesvorný, D., \& Vokrouhlický, D. 2008, AJ, 136, 291

Rubincam, D. P. 2000, Icarus, 148, 2

Spearman, C. 1904, Am. J. Psychol., 17, 72

Taylor, P. A., Margot, J.-L., Vokrouhlický, D., et al. 2007, Science, 316, 274

Čapek, D., \& Vokrouhlický, D. 2004, Icarus, 172, 526

Durech, J. 2002, Icarus, 159, 192

Vokrouhlický, D., \& Čapek, D. 2002, Icarus, 159, 449

Vokrouhlický, D., Nesvorný, D., \& Bottke, W. F. 2003, Nature, 425, 147

Vokrouhlický, D., Brož, M., Bottke, W. F., Nesvorný, D., \& Morbidelli, A. 2006, Icarus, 182,118 